\title{
Log-Euclidean distance based superpixel segmentation for PolSAR images
}

\author{
Hongyan Kang ${ }^{1, ~ a, ~ Y u e ~ Z h a n g ~}{ }^{1, b}$, Huanxin Zou ${ }^{1,} \mathrm{c}^{\star}$ and Tiancheng Luo ${ }^{1, d}$ \\ ${ }^{1}$ College of Electronic Science and Engineering, National University of Defense Technology, \\ Changsha 410073, P.R.China;
}

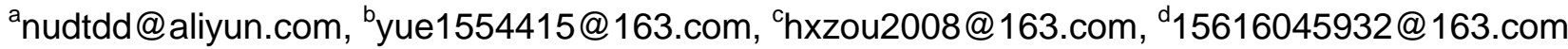

Keywords: SLIC, polarimetric synthetic aperture radar, Log-Euclidean distance, Postprocessing

Abstract. The simple linear iterative clustering (SLIC) method is a popular recently proposed superpixel algorithm for its simpleness and good performance for optical images. However, it may provide poor superpixels for polarimetric synthetic aperture radar (PolSAR) images because of the inherent speckle noise. In this paper, an improved SLIC based on Log-Euclidean distance with a novel postprocessing procedure by iteratively merging similar superpixels as well as preserving strong point targets is proposed. Experiments on a real image from ESAR demonstrate its superiority over two state-of-the-art algorithms, i.e., SLIC-GC and standard SLIC.

\section{Introduction}

Superpixels, which are clusters of pixels with similar color or some other low-level featuers, are finding more and more applications in image processing, such as segmentation ${ }^{1}$, object tracking ${ }^{2}$, recognition $^{3}$ and change detection ${ }^{4}$. The term superpixel is firstly proposed by Ren and Malik ${ }^{5}$ in a graph segmentation algorithm called normalized cuts algorithm (Ncuts). Another graph-based algorithm named Entropy Rate Superpixels is proposed in the literature[6]. Besides the graph-based algorithms, clustering-based methods are also popular. Quick-shift ${ }^{7}$ clustering algorithm, which assigns each data point to its closest neighbor with respect to a kernel function, is one of clusteringbased algorithms proposed for superpixel segmentation. Another clustering-based popular algorithm is simple linear iterative clustering (SLIC) ${ }^{8}$ based on k-means clustering. A feature vector consisting of lab color values and positions of pixels is utilized in SLIC algorithm.

All the superpixel segmentation algorithms mentioned above are proposed for optical images, which usually are not disturbed by noise. If these algorithms are directly used to segment PolSAR images, the boundaries of generated superpixels will gravely deviate from the real edges and many small isolated regions will be generated, which is caused by the speckle noise in PolSAR images. In addition, there are many small-sized or slim regions in PolSAR images. In order to obtain the edges of these regions, the initial grid width should be set as a small value, which indicates that the expected superpixel number should be large, thus resulting in quantities of superpixels in the end. The greater the number of final superpixels is, the higher the computational complexity of its applications is. Therefore, it is also important to obtain as few superpixels as possible with good boundary adherence.

To address the challenges, an improved SLIC is proposed in this paper. Log_Euclidean distance is utilized instead of the lab Euclidean distance to overcome the effect of speckle noise. Two steps in postprocessing procedure are used to obtain as few superpixels as possible and to maintain good boundary adherence meanwhile. The first step is to remove the generated small isolated regions as well as to protect strong point targets. The second step is to merge similar superpixels iteratively until a certain stopping condition is reached.

\section{Standard SLIC}

SLIC has shown good performance for optical images. In Local k-means clustering, each pixel is assigned to the closest cluster whose searching area covers its location. Let $\left(l_{i}, a_{i}, b_{i}\right)$ be the lab data of $i$ th cluster center with position $\left(x_{i}, y_{i}\right)$ and $\left(l_{j}, a_{j}, b_{j}\right)$ be the lab data of the $j$ th pixel with 
the position $\left(x_{j}, y_{j}\right)$. Then the combined distance as defined in Eq. 1 is utilized to determine the closest cluster center.

$$
D(i, j)=\left(d_{c}(i, j) / m\right)^{2}+\left(d_{s}(i, j) / S\right)^{2} .
$$

where $d_{c}(i, j)=\sqrt{\left(l_{i}-l_{j}\right)^{2}+\left(a_{i}-a_{j}\right)^{2}+\left(b_{i}-b_{j}\right)^{2}}$ is the data distance between the cluster center and the pixel and $d_{s}(i, j)=\sqrt{\left(x_{i}-x_{j}\right)^{2}+\left(y_{i}-y_{j}\right)^{2}}$ is the spatial distance between them.

\section{Proposed Superpixel Segmentation Algorithm}

Log_Euclidean Distance. Generally, a pixel of PolSAR data can be represented as a matrix called Sinclair matrix, denoted by

$$
\mathbf{S}=\left[\begin{array}{ll}
S_{H H} & S_{H V} \\
S_{V H} & S_{V V}
\end{array}\right] .
$$

where $\mathrm{S}_{H V}=S_{V H}$. The lexicographic scattering vector $\vec{k}_{l}$ can be denoted by

$$
\overrightarrow{k_{l}}=\left[\begin{array}{lll}
S_{H H} & \sqrt{2} S_{H V} & S_{V V}
\end{array}\right]^{T} .
$$

where the superscript $T$ denotes the transpose of a matrix. In practice, the covariance matrix $\mathbf{C}$ or coherency matrix $\mathbf{T}$ is used on behalf of a pixel. The covariance matrix can be defined by the outer product of $\overrightarrow{k_{l}}$ as follows

$$
\mathbf{C}=\frac{1}{n} \sum_{i=1}^{N} \overrightarrow{k_{l}} \vec{k}_{l}^{H}
$$

where $H$ denotes the conjugate transpose of a matrix, and $\mathrm{n}$ represents the number of looks. We can demonstrate that $\mathbf{C}$ is Hermitian positive semidefinite. For $\forall x$,

$$
\mathbf{x}^{H} \mathbf{C x}=\sum_{i=1}^{n}\left({\overrightarrow{k_{l}^{H}}}^{H}\right)^{H}{\overrightarrow{k_{l}^{H}}}^{H} \mathbf{x} \geq 0
$$

Therefore, $\mathbf{C}$ can be mapped to Euclidean space using the matrix Log function, which makes it possible to be called Log-Euclidean metric ${ }^{9}$. The Log-Euclidean (LE) distance ${ }^{10}$ between two covariance matrixes $\mathbf{C}_{1}$ and $\mathbf{C}_{2}$ can be defined as

$$
D_{L E}\left(\mathbf{C}_{1}, \mathbf{C}_{2}\right)=\left\|\log \left(\mathbf{C}_{1}-\mathbf{C}_{2}\right)\right\| .
$$

where $\|$.$\| means the Euclidean norm. The Log-Euclidean mean on a set of covariance matrixes can$ be obtained by

$$
M_{L E}=\operatorname{Exp}\left(\sum_{i=1}^{n} \log \left(\mathbf{C}_{i}\right)\right) .
$$

It can be rewritten in a recursive form ${ }^{10}$ as Eq. 8 for more efficient computation .

$$
\mathbf{M}_{n+1}=\operatorname{Exp}\left(\frac{n}{n+1} \log \left(\mathbf{M}_{n}\right)+\frac{1}{n+1} \log \left(\mathbf{C}_{n+1}\right)\right) \text {. }
$$

where $\mathbf{M}_{n}$ is the LE mean for the first $n$ covariance matrixes. $\mathbf{C}_{n+1}$ is the new covariance matrix for the $(n+1)$ th iteration. 
In this paper, Log-Euclidean distance is employed as the data distance in the Eq.1 and Eq.8 is utilized to compute the corresponding cluster mean.

Postpocessing. In PolSAR images, the small superpixels, which may be largely different from their neighborhood, are called strong point targets. If CCA is used as the postprocessing procedure, many strong point targets may be merged, thus leading to poor boundary adherence. Therefore, the strong point targets must be preserved. In addition, fewer superpixels should be generated in the end in order to reduce the computation complexity of its applications. To these problems, a novel postprocessing procedure consisting of two steps based on dissimilarity measure is proposed in this paper. The dissimilarity between two superpixels with the LE mean can be calculated by

$$
G\left(R_{i}, R_{j}\right)=\frac{1}{q}\left\|\frac{\mathbf{C}_{i}^{\text {diag }}-\mathbf{C}_{j}^{\text {diag }}}{\mathbf{C}_{i}^{\text {diag }}+\mathbf{C}_{j}^{\text {diag }}}\right\|_{1}
$$

where $q$ is the dimension of the vector $\vec{k}_{l}$, and $\mathbf{C}^{\text {diag }}$ is a vector composed of the diagonal elements of covariance matrix of a cluster center. $\|\cdot\|_{1}$ is 1 -norm.

The first step of the postprocessing procedure is to remove small isolated regions, whose size is smaller than a threshold $N_{t h}=S^{2} / 4$, as well as to maintain strong point targets. If the smallest dissimilarity between a small superpixel and its neighbors is smaller than $D_{t h 1}$, merge the superpixel into the neighbor with smallest dissimilarity between them. If not, move to the next superpixel. The second step is to iteratively merge regions with size larger than $N_{t h}=S^{2} / 4$ until the fraction of the merged superpixels and original superpixels in an iteration is smaller than a small value $P_{t h}$. In the second step, another threshold of dissimilarity $D_{t h 2}$ is needed to determine whether the superpixels are merged into the neighbors or not. An 8-neighborhood is utilized in both of the steps.

\section{Experiments and Discussion}

To evaluate the performance of the proposed method, a real PolSAR data set from ESAR is selected for comparison experiments of standard SLIC, SLIC-GC ${ }^{11}$, and the proposed algorithm.

The real PolSAR data set is an ESAR L-band PolSAR image from the Oberpfaffenhofen test site, Germany. A preprocessing of six looks in the azimuth and three looks in the range were performed to equalize the resolutions of the azimuth and the range for the sake of the facility of visual interpretation and evaluation. So the size of the second PolSAR image is $469 \times 513$ after the multilook processing. $D_{t h 1}=0.3$ by experience for SLIC-GC and the proposed method, $D_{t h 2}=0.12$, and $P_{t h}=0.02$ for the proposed method in all experiments.

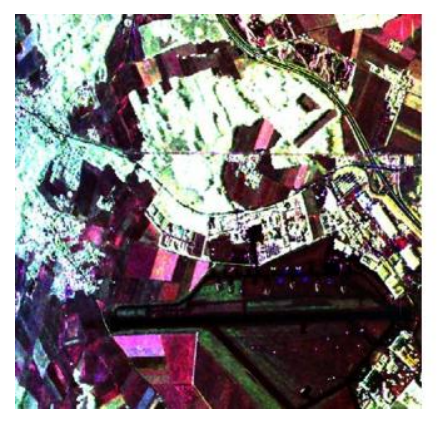

(a)

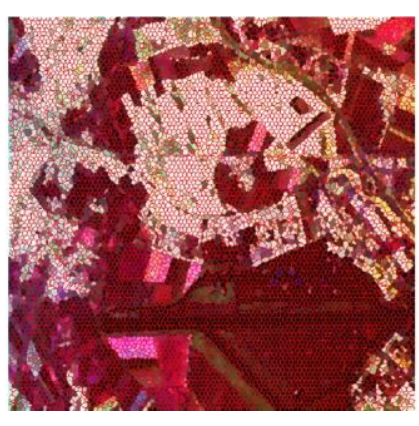

(b)

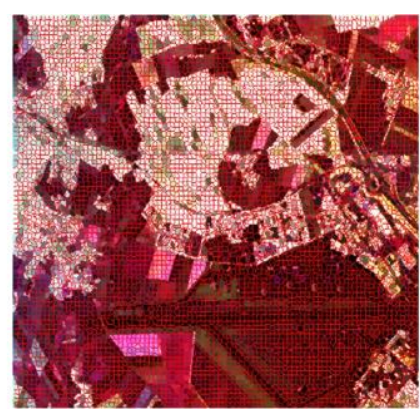

(c)

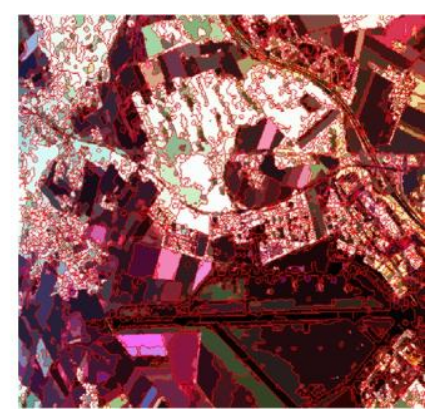

(d)

Fig.2. the results of three algorithms based on the real POLSAR image. The initial grid width $S=5$ for the image. (a) the Sinclair RGB image; (b) the superpixels with the number 8957 generated by standard SLIC; (c) the superpixels with the number 10259 generated by SLIC-GC; (d) the suppixels with the number 3001 generated by our proposed method. 
To evaluate the proposed method, experiments of the three methods on the real PolSAR image were conducted. The compactness $m$ was set as 15 for SLIC, 0.3 for SLIC-GC, and 0.8 for our proposed method. The results are shown in Fig.2. It can be seen that our proposed method outperforms standard SLIC and is comparable to SLIC-GC in boundary adherence with about one third of number of final superpixels yielded by standard SLIC and SLIC-GC. Therefore, our proposed method performs well in superpixels' generation with much fewer superpixels.

\section{Summary}

SLIC is originally designed for optical images and it can not be directly applied to PolSAR images because of the effect of inherent speckle noise in PolSAR image. Therefore, an improved SLIC utilizing Log-Euclidean distance instead of lab Euclidean distance as data distance measure was proposed to overcome the influence of speckle noise. In order to obtain as few suerpixels as possible to reduce the computation complexity of superpixels' applications, a novel postprocessing procedure by iteratively merging similar superpixels as well as preserving strong point targets is proposed. Experiments on a real PolSAR image demonstrated the effectiveness of our proposed method.

\section{References}

[1] P. Arbelaez, M. Maire, C. C. Fowlkes, J. Malik, From contours to regions: An empirical evaluation. In CVPR (2009) 2294-2301.

[2] S. Wang, H. Lu, F. Yang, M. H. Yang, Superpixel tracking. In ICCV. (2011) 1323-1330.

[3] J. P. Kaufhold, R. Collins, A. Hoogs, P. Rondot, Recognition and segmentation of scene content using region-based classification. In ICPR. 1 (2006) 755-760.

[4] M. Gong, L. Su, M. Jia, et al., Fuzzy clustering with a modified MRF energy function for change detection in synthetic aperture radar images, IEEE Transactions on Fuzzy Systems, (2014) 22(1), 98-109.

[5] X. Ren and J. Malik, Learning a classification model for segmentation, Proc. 9th IEEE ICCV, (2003) 10-17.

[6] M. Y. Liu, O. Tuzel, S. Ramalingam, et al., Entropy rate superpixel segmentation, 2011 IEEE Conf. on Computer Vision and Pattern Recognition (CVPR). (2011) 2097-2104.

[7] A. Vedaldi, S. Soatto, Quick shift and kernel methods for mode seeking, European Conf. Computer Vision, ECCV. (2008) 705-718.

[8] R. Achanta, A. Shaji, K. Smith, A. Lucchi, P. Fua, S. Susstrunk, SLIC superpixels compared to state-of-the-art superpixel methods, IEEE Trans. Pattern Anal. Machine Intell. (2012) 34(11) 22742281.

[9] V. Arsigny, P. Fillard, X. Pennec, N. Ayache, Log-Euclidean metrics for fast and simple calculus on diffusion tensors. Magn Reson Med. (2006) 56: 411-421.

[10] G. Cheng, H. Salehian, B. C. Vemuri, Efficient recursive algorithms for computing the mean diffusion tensor and applications to DTI segmentation, European Conference on Computer Vision. Springer Berlin Heidelberg, (2012) 390-401.

[11] F. Qin, J. Guo, F. Lang, Superpixel segmentation for polarimetric SAR imagery using local iterative clustering, J. IEEE Geoscience and Remote Sensing Letters, (2015) 12(1): 13-17. 